\title{
Das Gumscis,
}

urm

Prorector Dr. $\mathfrak{T} \cup y \mathfrak{e r}$ in Minten,

Ebrenmitalieb bes setrint.

Beatus qui poluit rerum cognoscere causas!

Das Grandecis ift cine fo befante, fo leidit zu erplas

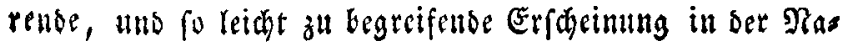

falls bie (Segenwart von $\mathfrak{B}$ rom im $\mathfrak{B a f f e t}$ beb tobten Miers nadbewiefen (Poggendorf's Annalen VIII. 475). Die trefflidbe Unterfuchung bes $\mathfrak{x a f f e r}$ vom tobter

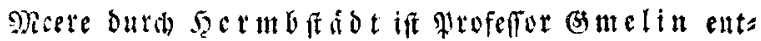
gegen. Sie findet fith in Schweigger's Jonrnal XXXIV. 177. Shad biefer enthalten 100 Theile bez saaffers:

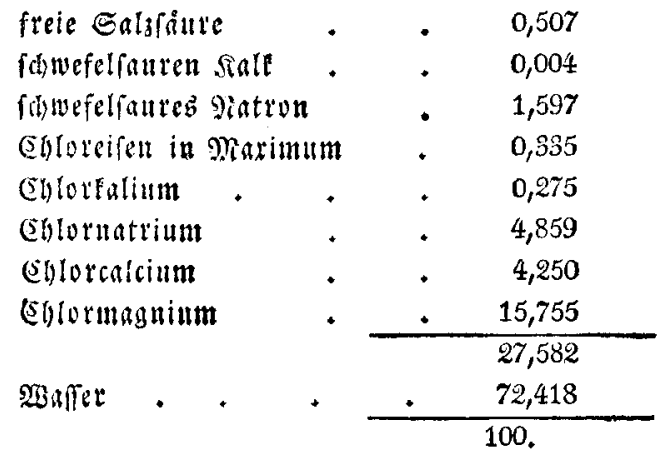

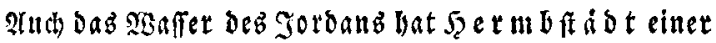
genanen analyje unterworfen. Es beftand in 10,000 $\mathfrak{E l}$. aนเ :

\begin{tabular}{|c|c|c|}
\hline (d) wefelfautem $s$ & & - \\
\hline Ci)lornatron & - & + \\
\hline Egforcalcium & + & - \\
\hline (5)lormagnium & . & . \\
\hline
\end{tabular}

Swwefelwafferfoff: Spuren

Misaffer

$+\quad 9950$ 
sur, Das es wirplid nid)t z" begreifen, wie man fo lange Dariber in Ulngewişbeit fenn, ja wie eb einige fúr unwabrs faeinlid baben balten, nod mebr, wie andere bie offenbare Realitat biefer Erfoleinung baben lingmen posnen, um fo mebr, oa wir jeft in Mbjut biefer Erfdeinung burd bas Bengnifa ber attogezeidneteften Maturforfder einez $B$ effern berelit find, unb baourch allein fdon bie Gadje, ibrer Ers fheinung und, aller dlien zweifer gefertift. So erziglt

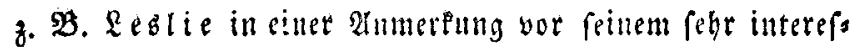

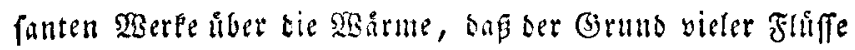
in ber Saweiz uno Sibirien, wäbrend oem grojten Theile

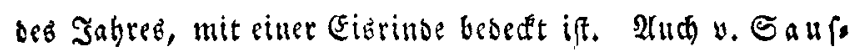
fure erwabnt einer ábulichen Erideinung im Benfer, See u. a. m. Die Erfdrinung iff alfo unlångbar gewiê, fo unnogidid fie aud idseint, uno fo fefre fie den befanuten

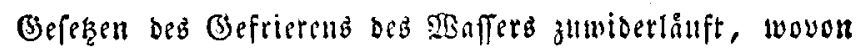
inbeffen, to viel mir befannt, bie Urfaden nod nidht erơrs tert fino. Ebe id mich jeood bierauf einlaffe, wollen wir die Sadhe, ibrem eriten Entftegen nah, verfolgen, die vers fäisenen Meinungen darbiber wairbigen, unb fobann unfere eigenen 2 nfidten onriber mittlyeilen.

(5. ift befaunt, dap verfhicbene Finffe, bod nidht alle, bei einem gewiffen Grabe von falte, mit treibendem Eife, bas man (j) $r$ unbei neunt, fid bebecten. Die Flúffe,

Moraus bervorgebt, baf bas Saffer bez Jorbanz yon bem deb todten Miets werentlich verfoleden fey (Rergl. oben (s) $m e l i n$ ).

Ein bituminofer salf aus ber Begend bes todten Meer entyielt Gohlenfinute 26,0, Sult 41,25, Ibon 3,71, Edwe: fel 0,90 , Aspbalt 13,39, צBaffer 13,75, und bie 3erglies

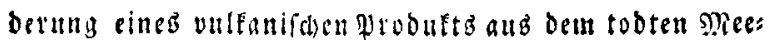
re ergab ferern $\mathfrak{f} e \mathfrak{r m b}$ fid ren Salk 3, Siefelerde 34, Eifendyd 21, Elonerbe 26,15, Naffer 10. $\mathfrak{X} r$. 
welde Diefe Erfideinung Darfellen, fino folde, orwen lifir bin wo wieber flad, uno mit grobem Riespande ooer 2 ratus den bebeft finb; dagegen in Fliffen, bie allentbalben r:ef fino, ersigte Hfer uno einen folammigten Boben babin,

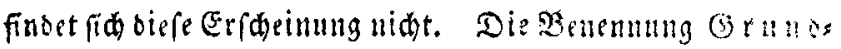
e $i$ b laft fhou feinen Urfprung vermutgen, uns matt bin: te ald biter fagen: vox populi, vox veritatis! Hub ted

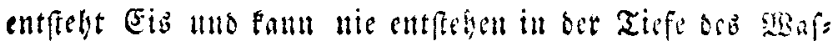
fers, fondern nur fetb nuf ber Sberflähe, uno auts sics, im frengfen Cinne genommen, nur bei rubigem nib nie

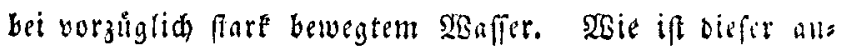
farinende 2Biserfprud ju liffen? -

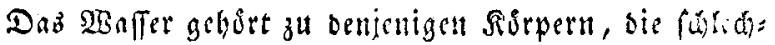
te feiter für bie Sabrme fino. Ea nimmt bie WBarme fibr langfam an, uno bauert baher tm fo langer, eine brous tenbe Menge zum Ruden zu bringen, je gröper fie iff, wes: balb grope uno tiefe Deftillirgefiafe nahtbeilig, je fladice bagegen, defto vortbeithafter finb. Itmgefebrt vertart eine fodende Flifingleit befio langfamer ben $2 B$ armefoff, je bes beutender je tiefer, uno in je weniger leitenden Bififien, etwa felz, fie ift, unb alth bann nur immer alt ber Sbes" flade. Soll baber bas warme 2 Baffer fonell feinen sears meftoff verlieren, fo muß es in flade, ftarf leitende, stwa metalletle Gefäke gebradt werben, bamit ilgm babuth bet TSarmeftoff yon allen Seiten forme entzogen werben; batai

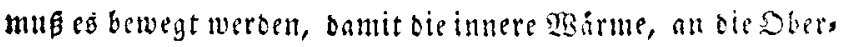
fladbe gebratjt, ifm fo yon ber umgebenden faltern Rlift cm: jogen werbe.

Dieb viffeno, unb alp befannt vorangefert, Fonnten

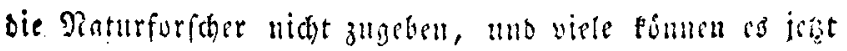

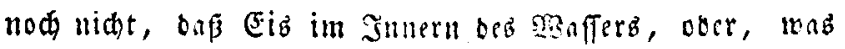
bier baffelbe ift, auf bem Grunbe beffelben fid bilbe oder entfeben fsune. Da a i a nenut biefe Joee fogar ein ge" 
neinea $\mathfrak{B}$ orurtheil ber Fifder. Die Erfaheinuth bes Zreib. ober Girubetfeb iff unu aber einmal ba, fie whïten fich alfo nidt anders zu belfen, als zu fagen: $\mathfrak{e s}$ falle in $\mathfrak{N a s}$

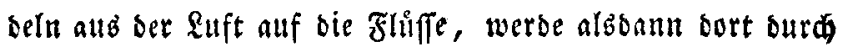
ben Froft weiter ausgebilbet, immer gröser uno gróser, bis

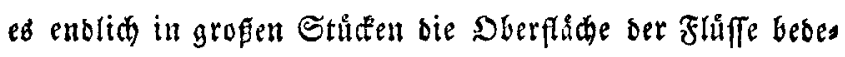

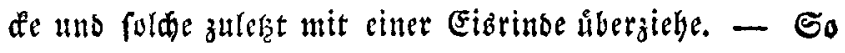
naturlid biefe Erflarung ift uns fo leidt man bies zuges ben founte, fo mikte oo der jedesmarigen Formation bes Eifeś auf ben Fluffen, baв Dafeyn biefer Eiżnabeln in der Iuft, alb vorbergebenb, bewiefen werben, wab nidt nut nidat moglid, fonbern rogar gegen bie Erfabrung ift; benn

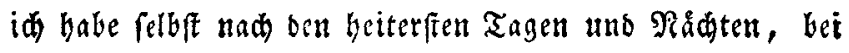

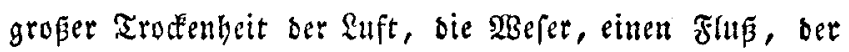
bie Erfheinumg bes Grunbeifes am meiften barbietet uns

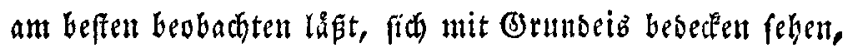
und jene Fionabeln finben fich bod nur bei neblidter, fettds= ter $\&$ uft, wodurch der fogenanute Rauhfroft an $\mathfrak{B a}$ amen entfteht. Çs ift alfo diefe Bebauptung wo nidht nidbtig, bod burchas umzuláfíg.

Dagegen bebaupten nutn andere, j. $\mathfrak{B}$. Nollet, bab

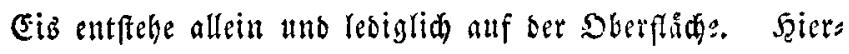
gegen läpt fich nidbs einwenden, vornubefert, baj bas Baffer rubig ftebe, uno feime Suerflathe unbervegt, oser Dod) nur måjig bewegt fey. Dem wirb die Sberfadde

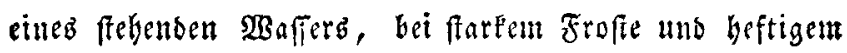
Stturme, fo zu fagett, surbetuandergefdingen ober ges peitfdt, fo entfelbt allerbings Eis, abce nie ein fpiegels glatteb, fonbern cin 2 linttereis, dab sann bie Sberflade wie Berge uno Thille: bedect, je nadjocm ben $\mathfrak{B e l l e n}$ ber WBarmeftoff entzogell wurbe, wodurd fie ju Eis erfarrten. Diefes Eis ift, fo zu fagen, nidt auf dem sisaffer, fonoern in oer $Q_{\text {uft }}$ entfanden, weil butd bab fortwabrente Alifs 
whiblen bes 2Raffers bura ben Gturm bie Ientperntur tes

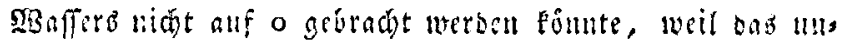
tere winere mit oem obcren fisteren fortuabrens 3 th einer bsheren Temperatur, alfo, fid mirchen unb basurd niche

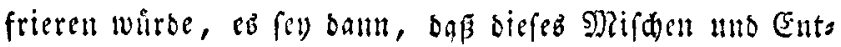
zirhen bes şarmeftoffeb fo lange fortgefert wirbe, bio bas galije SSaffer jur Remperatur o gebradt uns baburd it

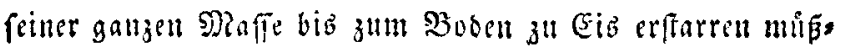
te. Eime biefer ålntidbe Erfdeinung crfolgt, wenn man cine Sdaale mit $\mathfrak{B} a f f e r$, bie rubig in einem verfdloffenen Simmer fand, bas uad unb uad weit unter bie Temperas tur o gelangte, babei nidt gefror, nun profecid antilgrt, uns baburd mit einem Male in Bláttereib in reinen ganjen Maffen verwanbelt witb.

Wie febr bas $\mathfrak{T B a f f e r}$ librigens mit bem Bämeftoffe yerwanst fey, unb wie folver es inn fabren laffe, davon ift

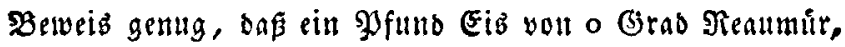
benn mebr entyelt ez nie, cin $\mathfrak{Y}$ funb $\mathfrak{B a f f e r}$ you $+62^{\circ} \mathfrak{R}$. erforbere, um in Şaffer von ber Temperatur o verwandelt

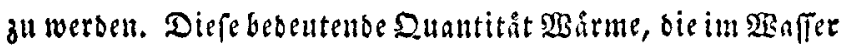
in gebunbenen 3 slftanbe ift, muß eв bod) erft vertieren, went eb in Eits folf verwanbelt werbent. Daburd hålt es fo fduer, einen fark beluegten Flup mit (5iš ju besecten, weil bas untere warmere Șaffer fid fortwabtend mit bem oferen falt gewordencm wieber vermijdt uns fo bas Jeft.

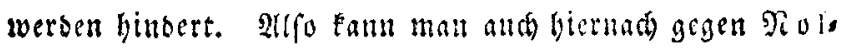

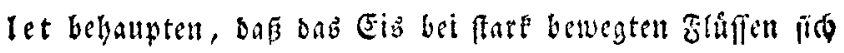
nidat auf ber Sberfind berfelben bilocn fintte.

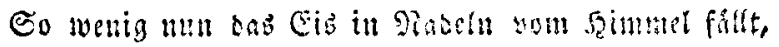

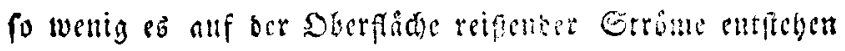

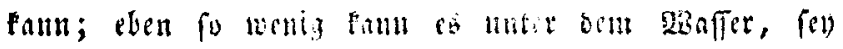
es in ber Mitte over auf bem Şosen ocfielorn, sutfeflen, XXII 28. 1 sift. 
Dem wie follte ibm ba ber Sa ármeftoff entjogen werben fin. nen, oljne welches eb nide fift werben pann? - Span bat

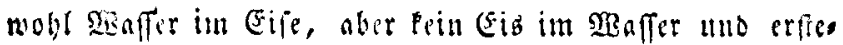
res alld nur in fliturn bringen, wo ifm son allen Eitin

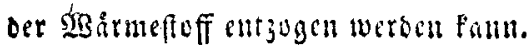

Da mun nber nidet eimmal gelitugnet werben fam, oaß es Brmbeis und Eib auf bem Branbe ber Soen unb Filffe, alfo Eis unte: bem seaffer giebr, wovon nadber, fo fino Etuige allf ben Groanfen zefommen, bas $2 B a f f e r$ verticte

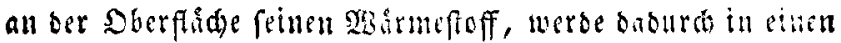
engeren Raum gebracht, nub bermad fpecififd fotuerer, fo

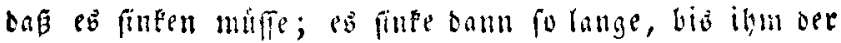

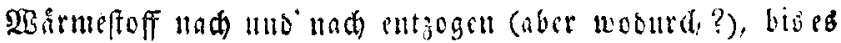
z" Eis geworoen, wieberam surd feime fpeciffere fridtigs

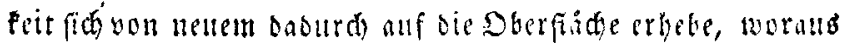

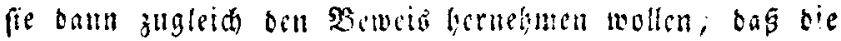
Tomperatur in ber Tiefe tes Saffers abuchime, was gegen alle Befere ber Natur uno jugleid gegen alle Erfabrumg ift; benn wenn igm nur ber sisermeftoff an ber Sberfäde ents zoget werben fann, was fell es bann nud in ber Tiefe thun, wo, weun es bort fo falt ware, fid yon felbft fobon Fis bitben unb allf bie Sherflihe fteigen wurbe, olye baj bab Saffer von oben nad unten zu Fommen braudte; ift bieb aber niate der Fall, fo wirb audh bier wieber bem von

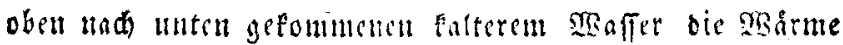
mitgetfeilt, uno es basurd nidt zu Eis werben fönmen. Und allerbem, wer bat lod) je gchirt, bas sisaffer im Baffer fower foy? Die Temperatur beb Bafo fers, fowie oer \&uft, uimmt thbrigens wit ber Tiefe ju, wie eg nah ben Beferen der Edwe?

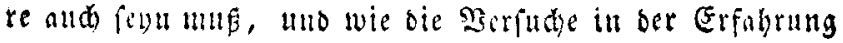
es bepatigrn. Fejren wir biefe Beweife. 
(5) ift sutd Barometer, und Thermometerverfuche ans: gemndt, oap die Ruft in bem SRaape fich veroume unb fálter werbe, ie melyr man fich son ber Groe entfernt, unb ungefebrt defto bifiter unb wåner werde, je mehr man fit bem SRittelpunpte der Eroe mabert. So fodt bas Taffer an ber Soberfádhe der Erbe unb bålt bei einem Barometers fanbe yon 337 framjofifden Sinien so Brab Reaumit. Dagegen allf oem Mont Nofa bielt bas pochende Saffer

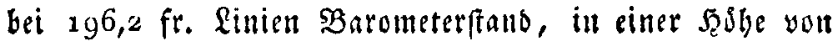
2312 Toifen, nut 68,4 Bras Reaumir. So foll nach

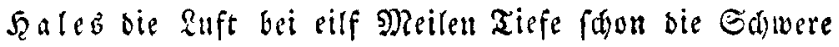
Deฉ̉ Golbez baben, uno wáre biez allh nidht ber Fall, to hat bod Sant gewis Nedt, weun er bied amimmt ant MittelpunEte ber Eroe. Fermer, fergt man unter die \&ufts pumpe eill Gefáp mit $\mathfrak{B a f f e r}$, und ein anberé barunter mit Sdweferfäure, fo wirb oas yon ber Ruft verdunfete

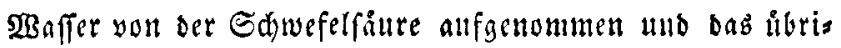
ge $\mathfrak{Z B} a f f e r$ in Fis verwanbelt. (Fine 5ofad) verbinnte \&uft bringt allf diefe SBeife so bis 100 Grab Ralte hervor.

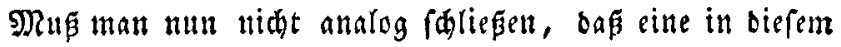

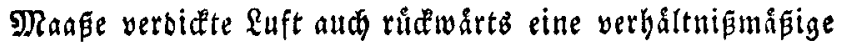
ŞBame erzellgen múffe? ausigemadot.

Fox fanb, bei wieberbolten $\mathfrak{B e r f u d e n , ~ i n ~ e n g l i f q e n ~}$ Gruben, in ber Tiefe von: 


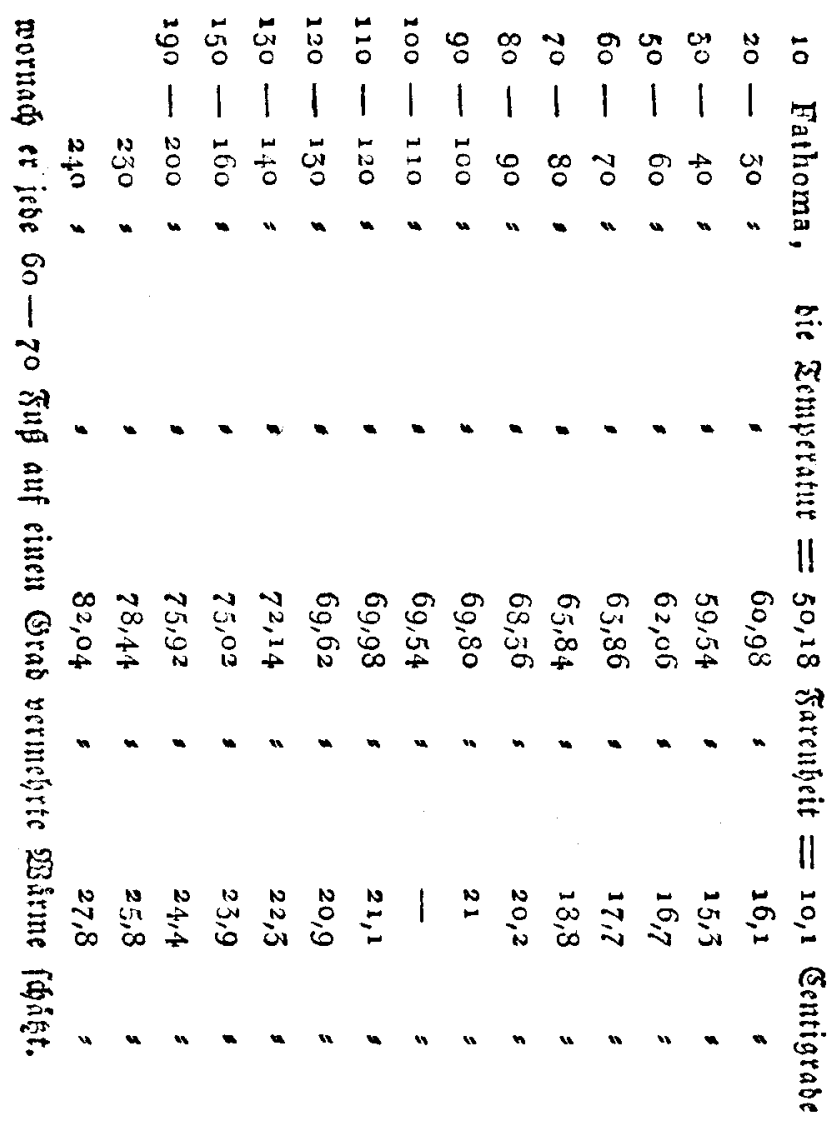

So bat aub $\mathfrak{D} \mathfrak{a} \mathfrak{u} \mathfrak{b} \mathfrak{i}$ ifou ourd Sarometerverfude

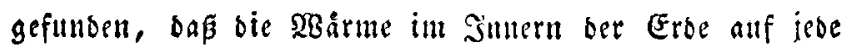
s80 Fn un einen Gras Seaumir zunebme, eine Sed)=

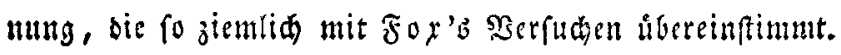

Reueroings hat Dr. Forbes biefen Gegenftand yon nettem vorgenommen, und in sen Bergwerén von Eormwall bie mittlere Temperatur ber luft uno ses 2 affers gefuls ben bei: 


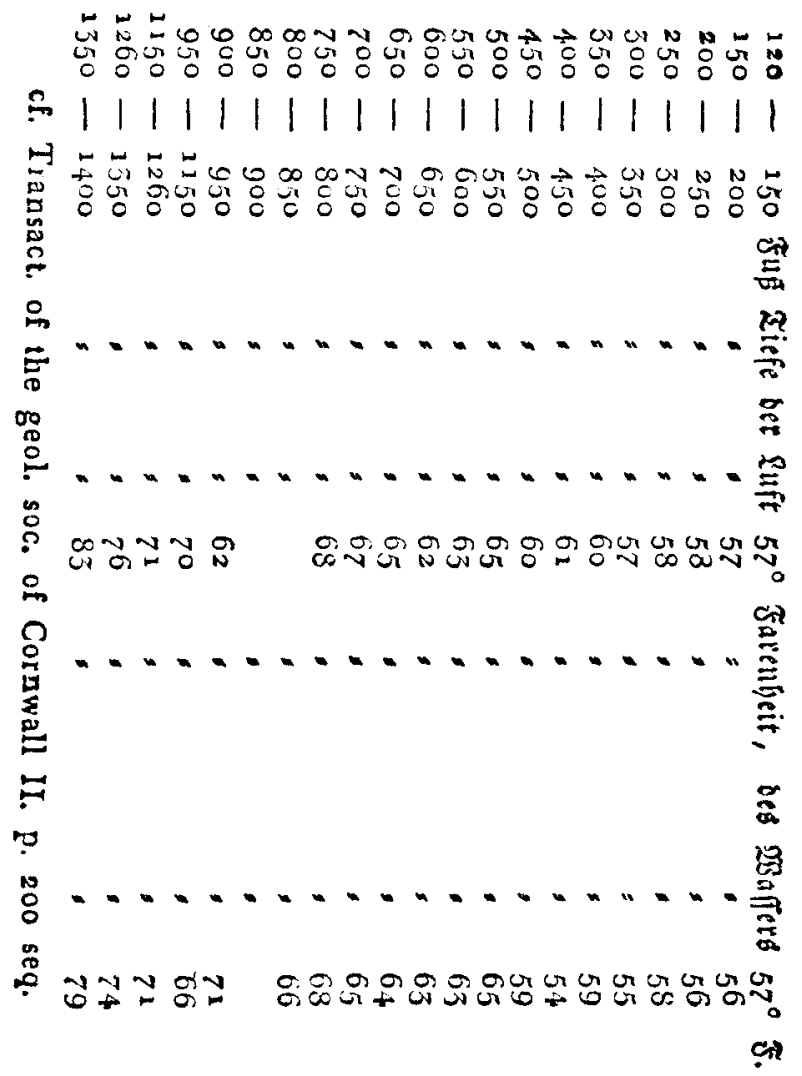

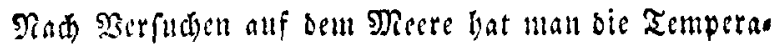

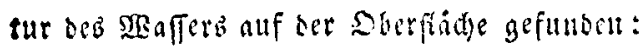

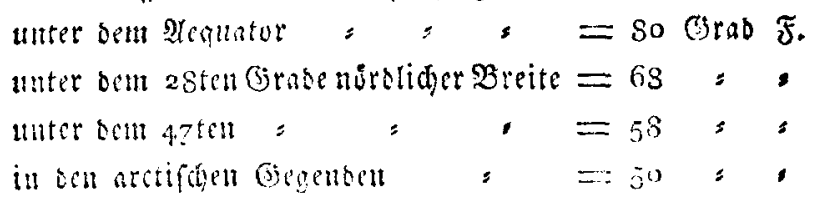

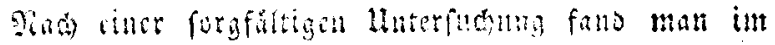

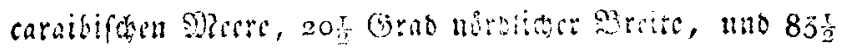

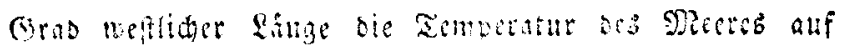

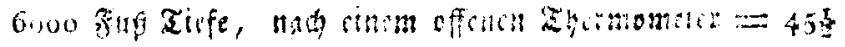


(5) rao, und nac cinem andern, ber in einem mefingenen Enlinder werichloffon war $=49 \frac{1}{2}$ Grab Farenheit. Die Derrfache bes suaffers war $82 \frac{\mathrm{T}}{2}-83 \frac{\mathrm{T}}{2}$ Grab.

Siad dichn, fo wie nad mebreren onriber angeffellten Berfuden, fanu man folgende Sabe ald allgemein ridutig antebmen: 1) Die Temperstur bes Seeans versingert fich

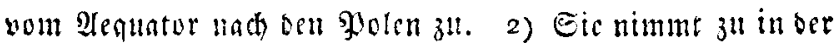

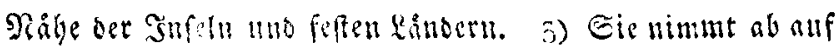
bem Scean mit juntymender Tiefe, in sen Wolargegenden Dagegen umgefelyt. 4) Sie if hber Sanbuinten geringer.

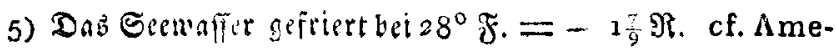
rican. Journ. Vol. V. n. 1.

Ad 3) Daj bie Mañme bes Decans unter sem Requas tor mit junebuener Tiefe, o. \%. To weit man ed hat uns

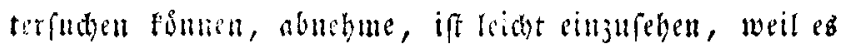
an ber Soberflabe, ourdh bie fiete Solmenwarme, weit fiber frine gewóbntide Semperatur enbughet wirs, uno alfo in ber Ilefe, wobin julle sidyt mebr wirfe, fid ber erferm wieber

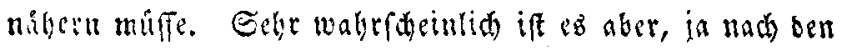
Geferen oer Sdwere nothwendig, bak oie armperatur all burt wieber jullehme, wemu fie riffwartz you oben sab Minimum erreichr bat, alb wolyin bie खsarme der Conne nicht melir oringen, uno wegen bortiger abfoluter Silbe bab Uuterfe mit scu Suerfen fid nide melye mifden Panu, wel, web benn auth sie Serfude in ben Molargegenden, wo

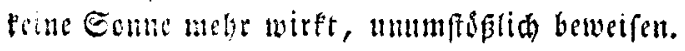

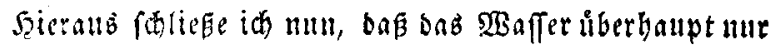
flifig fey ourd nic intere sirme oer Eroe, fie mag berfoms men, wober fie immer wolle, obne welches bab gBaffer, wes nigftens an ben Polen, ftets bis zum (5)runde exfarrt fenn

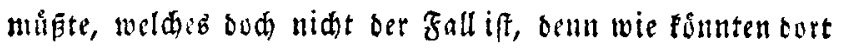
ISallfiche und anbere Sethiere leben? unb baber alth auf

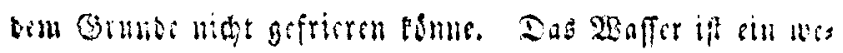




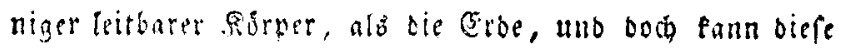

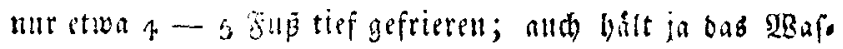
fer, fatbe in frengfen asiuter, unter oer Eroe, in ticfen Brumen now 8 - 20 Grab Sieammir, woralis zur Gies Mige burworgets, baj bas Sarter, fo wie bie Eroe, nie you unren math oben, fonbern ftets you Tage ab nad innen ge,

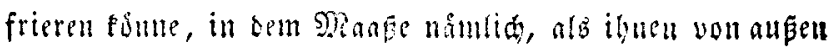

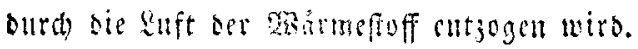

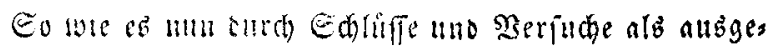

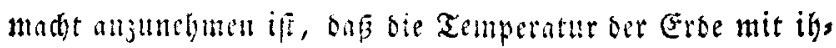

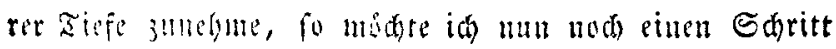
meiter gebell, uno betauptert, sap bas Smmere oer Erbe, um

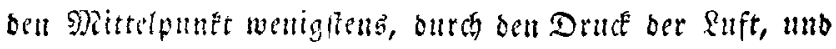
bie frets wadyfube Sraft ter Echwere, in feter Bluth fen, unb Daher bie feucrpeienden Berge, bie Erobeben und die beifen Suellen ju erfliren fint, uno oaf fo wie vermige biefer imeren, surd bie Sdwerfraft bewirten $\mathfrak{B a r m e , ~}$

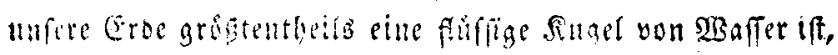
Der bei weitem grifiere, uno baourdh ourd bie Sdwerferaft nod mrbe comprimirte ßorper ber Eome, ebenfalls cine

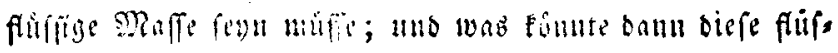

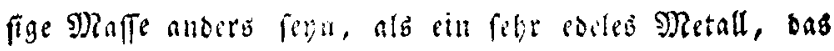

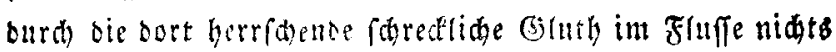

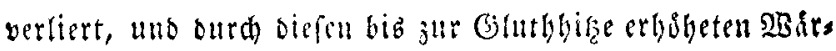
meftoff oas Ribt verbreiten mus. Soer fullten bie Befere ber Natur, etiva die Edyer Eraft nuf ber Somme anders

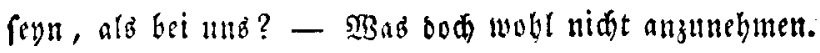

शadsent wir num gezeigt haben, onß fid (5is, weber

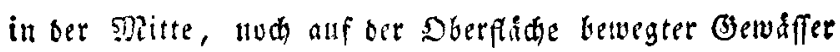
erjengen Esme, wno dub sie Erfalyrug biefer Theorie wi, orefpridt, ober wengltens ju witerfpechen fdeint, fobleibt

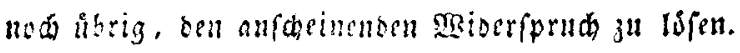

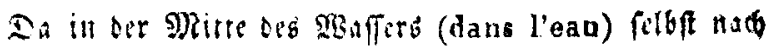


aller Erfabrung fid nie Eits erzengt, fo ift biefer Caks an fith flar. Das ater anf dem Grunde ser Een und Fluffe Eig vorfumme, brgengan mobrere glontwuirsige In\&uncr,

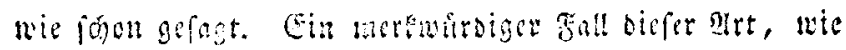
ibn Giarne in cinem oer nenerest Sefte seb Quarterly

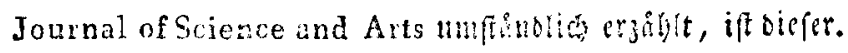

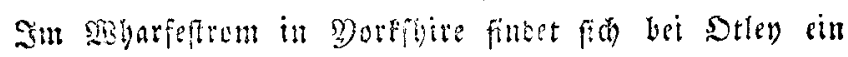
MBebr, weldes nus sidatcrifeinen qlleer sura ben Strom

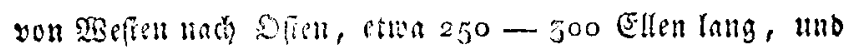
unter cittem xinfil von $35-50^{\circ}$ gegen Siorben gebaut

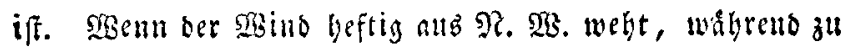
glether acit farker Froft eintritt, fo ilbergicben fich bie

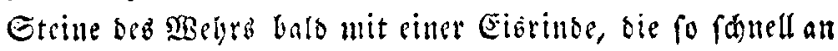
Dife zunimmt, dafi Eurze seit barauf ber lauf bed Stros

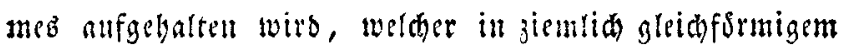

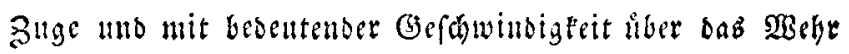
nieberfallt. NBeljt nun ber $\mathfrak{R}$. NB. $\mathfrak{B}$ Bitlo febr ftrenge, fo

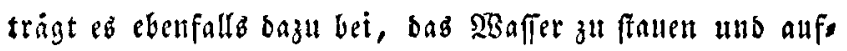
zuhalten, fo baj fich immer mebr Eis anfegt. Die Folge biervon iff, baf ber Strom in fllrzer seit formlid altfges dámmt uno oas șaffer oberbalb des $\mathfrak{X} B$ hyrs zll einem bó, bern Etande gebradt wirb, fo baf bie llfer haberfdwemmt erfdeinen. Dod) balo libt bas (Gewidht bes vielen SBaffers simen folden Drule gegen bab (Eib ans, baf bie fabwader

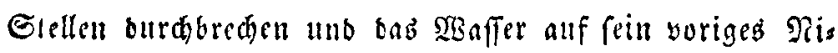
yaal gebradt wirs, worauf bann bas fonberbare Sdanfpiel

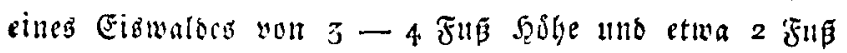

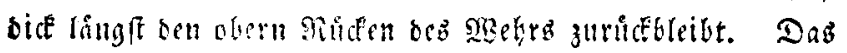
Eib ift unten von dichter Eonfiffell uno wur in ben oberen Sheilen alts eituer Menge biunter Gdeiben ober Lagen jus fommengefert, welche gams confus unter fehr yerfoledenen Sheigungawinteln ourd) cinanter gewablen find, wathrono

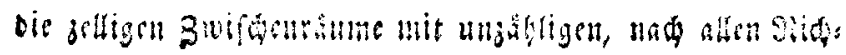


tungen fich burdirenjenben Eisfrablen befleibet erfecinen. cf. Annals of Philosophy 1822 March, on the formation of Ice in the Beds of Rivers by Th. M. Kegver.

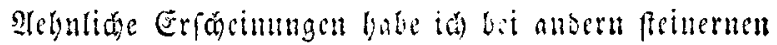
$23 \mathrm{ebren}$ wahrgenommen, nur nie bri bifjemen.

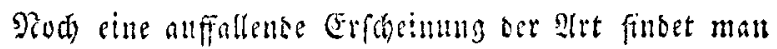

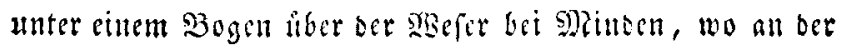
reifenofen Gtelle baz SBaffer nie friert unb ood oer Bos ben im SBaffer fid mit einer biden Etorinde, bei frrengem Frofte, überjiebt.

So fehr biefe Erfheinungen ber Theorie bez Befries

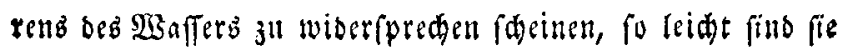
bod zu erfiaren.

Dab erfte, wab̉ man bei allengenannten zllerft finbet, fins

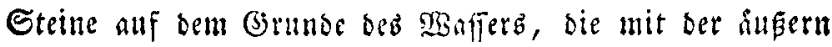
Euft in leitender Berbindung ftelli. Steine find leitbarere

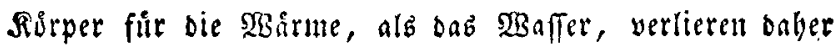

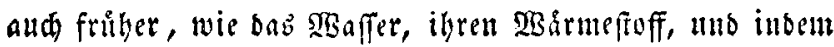
fie fo weit eher, alb das Saffer, unter Null fommel, ent, ziehen fie bem über ibnen fehenden ober liber fie binlaufen,

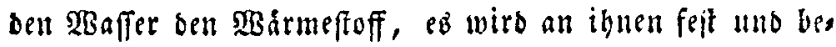

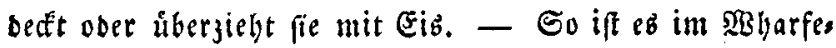
ftrome ber Fall, wo nad ber $\mathfrak{B e f d r e i b u n g ~ b e r ~} \mathfrak{R}$. $\mathfrak{B}$. $\mathfrak{R}$ ind

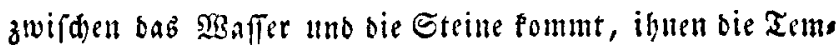
peratur der Suft mittbeilt und bas $\mathfrak{B a f f e r}$ baran erftarreu

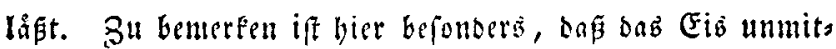
telbar an ben Gteinen bifter ift, alb weiter nads oben, weil ba bie Reitung ffitfer ift, als lier, wo bas liberfliefente Baffer fie bindert. So ift es berfille Fall in ber sejer

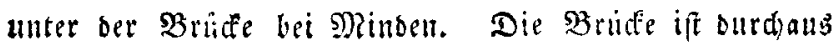
mafiity, unb rubet allf einem mafien Funoamente, bas uns

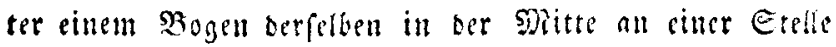

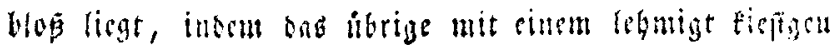


Buben bebeft ift. Die Eteimmoffe non aufen theilt num

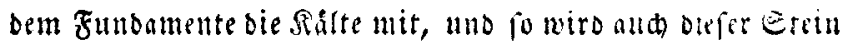
mit einer Eisrinbe beteft, obgleid bas 23affer bariber, felbft in ferengften Wiuter, ben 23. Sammar 1823, bei 23 (Grab Seanmir nidt gefror, da dod) die ganje $\mathfrak{B e f e r}$

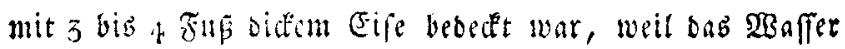
an biefer Stelle aum reisubften fliefit.

(5) cu fo blicb, Reobachtumgen zufolge, am SBinterfee, unter bem 6atcu Grad uordither Brsite, cf. Franklin's Journey to the Shores of the Polar Sea, im December 2822, bei einer Sirte von $-57^{\circ}$ Farenheit $=-39 \frac{5}{9}$

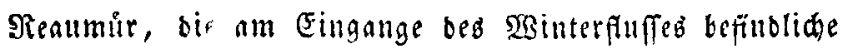
Stromfdnelle, felbft bet ber ftrengfen Ralte, offell, obwohl fie einigermagen an Breite verlor, wie ald bie zu Sins ben. Die Zemperatur bes :Raffera, fowobl bez offenen,

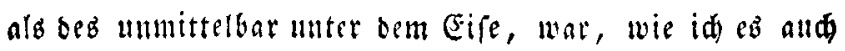
ftetb gefunben babe, $=32 \mathfrak{F}$. $=$ o $\mathfrak{A}$.; dent nie madht bas Fib eille grofare Rilte im $\mathfrak{B}$ affer, als $=0$. Die Tempes ratur bes̆ Sanfers inbeffon in einer Tiefe von $2 \frac{1}{2}$ Rfaftern war $=+42$ Farentrit $=449$ Sieammur, ein $\mathfrak{B e w e i s , ~ b a \beta ~}$ Das $\mathfrak{Z}$ affer ill ber Tife nidt gefrieren fann, wenn and bie

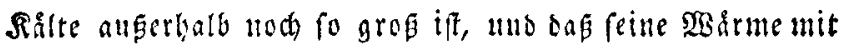
ber Tiefe zlunebme, felbft im fälteften Slima.

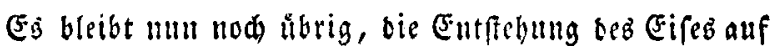
ber Dherffade ber Fliffe, a. h. bes Treibeifeb, ober bez fos genannten Grundeifes ju ertaren. Diefes Treibeis, wie roon gefagt, findet fich nut anf einigen ullo jwar allf fols Wen Fliffen, die flade unb ftcinige llfer baben. Die SBes fer iff befoubers ein folder Fluf. Isem Froft eintritt, fo fielt man bei niebrigerem ober beiboherem ssafiet sie Sber, flade berfelben fid mit eimem mebr ober meniger fommbis gen, broftichen Eife, bem $\mathfrak{B l i n t e r e i f e ~ a b n t i o h , ~ b o b e d e n , ~}$

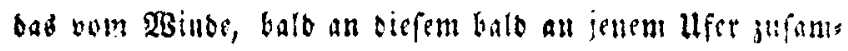


mengedrangt, fortgetrieben wird, unb ganz anberer Art ift,

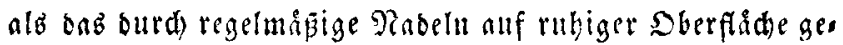
bilbete, unb babei mit eleinen Eteinchen ober Ries angsfillt iff, weldeb ben fidherften Beweis feiner Entfebung auf bem Grumbe liefert. Diefes Fib wito bit anbaltendem uno jus nebmendem Frofe immer fiater unb difer - crescit eundo - biš eş fị́) entitid an ocu fiahften Etellen ftautet,

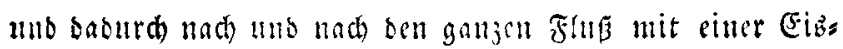
rinde bebect. Die feiniau uno fiejigten Hfer, bie babei fegr flad finb, verlieren bei weiten eher ben :Barmeftoff, als bas $\mathfrak{B a f f e r}$, uno entzielyen benfelben dem $\mathfrak{B}$ affer, felbft von unten auf einen Fup tiefen Safferb, bebeden ober übers zieben fich onourd mit ciner Frufte son Eis, uno weil biefe Steinden abgeriffen und locter liegen, werben fie bam letat burd bie Fisrinde geboben uno fhimimmen fort, wie id felbft mebrmals felyr genall beobadhtet babe. Dak bieb fo fey, beftatigt uod diefe (srfabrung. Sat namtid die Befer wenig $\mathfrak{B a f f e r}$, wobei oie fiefigtell Ufer blop liegen, fo bebarf ez nur eituer geringen Rialte, etwa 5 Orab Rcalts mur, unb das (5ib zeigt fid fofort bebeutend; ift aber bas

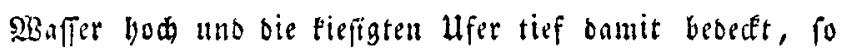
zeigt fich, felbft bei einer bedeutenden Salte, wenig oder gar fein Eib.

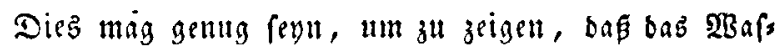
fer nie yom (brunde alts nach oben gefrieren fönne, weun nicht eitle leitenbe Subfanz ba ift, die bies betwirft. Es leibet biez die innere $2 B$ irme ber Erbe nidt, obne welde, fie mag nun berribgen, wober fie immer wolle, unier $\mathfrak{P l a}$ net groffentheils eill erftarter Eisflumpen, uno nidh wie jeg̨t, felbft in ben tiefî́n 2lbgrủnben bez Meeres̉ uno ber feften Erop, Der Sdauplake bez Drganismub uno bes thierifcen uno ve, getabilifden $\mathfrak{L e b e n b}$ peyn wurbe.

Den गuksen, ben man bieraus gieben finnte, wirb. 


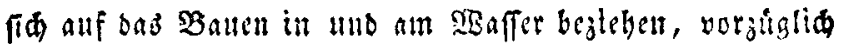
nuf bas Sdladten (fascinage) an reibenden Fllifen. Dieb follte nie gefolyen, alo bei ganj feidtem raffer; benn

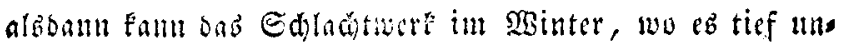

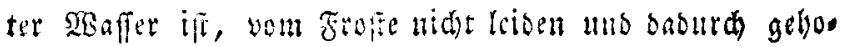
ben werben, wie es wobl gefditebt, befonters wenn bab Fledtuere mit Siles unb Steinen beitwert ift. In habe felbft erfahren, dak ein bedentendes Fledstwete, das als bes fene beftimmt war, bie SBefer an einer flachen Stelle zu sammen, um bas Faldrwaffer baneben für bie Shiffe tiefer

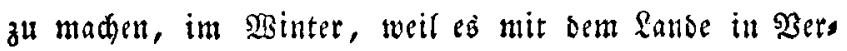
billong ftand, und mit Steinen unb Risbfanb befwwett war, burd unb burch fll (5ib gefroren, im abauwetter fid bob uns bavon fhwamm, woourd bebentende Foften zivect, Iob verloren gingell *).

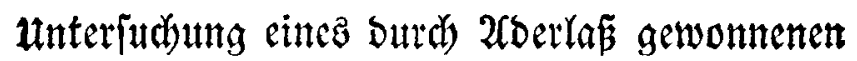

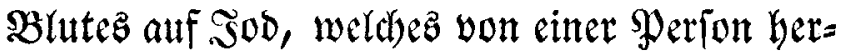
$\mathfrak{E a m}$, bie einige 3eit binourd) mit Soblatbe ein= getieben worden war,

von

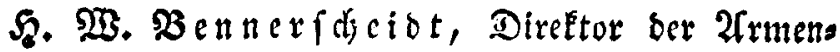
apotheke in $\mathfrak{2}$ adyen, streibdireftor des sereing.

Sn einem (serpriad, weldes id vor einiger zeit mit

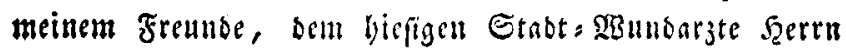

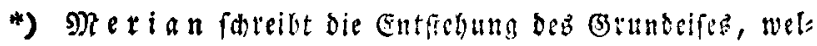
deb nie in febenden waffern crideint, ber Mifdumg

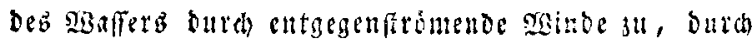
welche baz crealtete sisaffer ber soerfiade auf ben ormo bes gluffer lomme, unb an beh unebenbeiten sefietbru 